\title{
PRIMARY MALIGNANT MELANOMA UTERINE CERVIX
}

\author{
Asghar H. Asghar ${ }^{1}$, Sadia Rizvi ${ }^{1}$, Akhtar Ahmed ${ }^{1}$, Ahmed Nadeem Abbasi ${ }^{2}$, Abid Jamal ${ }^{3}$, \\ Javaid Mehboob ${ }^{1}$ \\ ${ }^{1}$ Department of Clinical Oncology, Karachi Institute of Radiotherapy and Nuclear Medicine, Karachi, ${ }^{2}$ Department \\ of Radiation Oncology, Aga Khan University Hospital, Karachi, ${ }^{3}$ Department of Surgery, Healthcare Hospital, \\ Karachi, Pakistan
}

Received: 8 June 2015 / Accepted: 31 January 2016

\begin{abstract}
A 40-year-old premenopausal female presented with foul-smelling per vaginal discharge for 3 months. Diagnostic work revealed a locally advanced primary malignant melanoma of uterine cervix. The patient declined pelvic surgery and was treated with Dacarbazine. Malignant melanoma is a rare tumour of skin and has been ranked in the top five cancers of Australia and Sweden. It is a tumour of melanocytes which forms melanin pigment in the skin. In men, the most common site is trunk while in females common site is limbs. However, melanoma can arise from mucosal surfaces where the melanocytes are present. Most common mucosal sites are head and neck followed by female genital tract.
\end{abstract}

Key words: Cervix, dacarbazine, malignant melanoma

\section{Introduction}

Malignant melanoma is a rare tumour of skin and has been ranked in the top five cancers of Australia and Sweden. ${ }^{[1]}$ Highest incidence has been reported from Queensland Australia. ${ }^{[2]}$ There is a slight preponderance of males as compared to female as reported in one study of Pakistan. ${ }^{[3]}$ Females most commonly develop these lesions on extremities while male usually on trunk and head and neck regions. No definite aetiology can be identified; however, it is found to be more in individuals exposed to sunlight. Interestingly, it does not have a direct relationship with the amount of sun exposure as is with other skin tumours because it is more common in white-collar workers than in those who work outdoors. Diagnosis is usually clinical, but histopathological confirmation is usually required. Special immunohistochemistry (IHC) stains, i.e., S-100, HMB-45, and Melan-A further helps in its diagnosis. The best treatment of melanoma is wide margin excision as it is radio and chemotherapy resistant. This middle age lady presented with unusual per vaginal foul-smelling discharge which on further workup was confirmed a case of primary malignant melanoma of cervix.

Correspondence: Dr. Asghar Hussain FCPS, Department of Clinical Oncology, Karachi Institute of Radiotherapy and Nuclear Medicine, Karachi, Pakistan.

Email: quaidian@yahoo.com

\section{Case Report}

A40-year-old premenopausal female presented with 3 months history of foul-smelling per vaginal discharge. She was housewife with no comorbidities. Her exposure to the sun was not extraordinary. She had seven children, all by spontaneous vaginal delivery. Her youngest child was 7 years old.

The patient was initially seen by local general physicians and was started antifungal treatment with no improvement in symptoms. She was finally seen by a gynaecologist and advised ultrasonography. Ultrasound revealed a $7.5 \mathrm{~cm}$ $\times 5.5 \mathrm{~cm}$ mass in a lower uterine segment involving the anterior wall of the cervix. The differential diagnosis was a calcified fibroid or a malignant growth. Per speculum examination revealed a growth in uterine cervix involving upper two-third of the vagina. Biopsy of the mass revealed primary malignant melanoma of the cervix. IHC was positive for S-100, HMB-45 and Melan-A while negative for cytokeratin AE1/AE3, cytokeratin 5/6, p-63, Desmin, CD-99, lymphocyte common antigen, synaptophysin and vimentin. Magnetic resonance imaging (MRI) pelvis showed $5.8 \mathrm{~cm} \times 8.0 \mathrm{~cm} \times 6.5 \mathrm{~cm}(\mathrm{AP} \times \mathrm{TS} \times \mathrm{CC})$ mass involving proximal two-third of the vagina and the distal part of the cervix. The mass was extending into endocervix and endometrium causing its thickening. Anteriorly, it was causing an indentation on the posterior wall of the urinary 


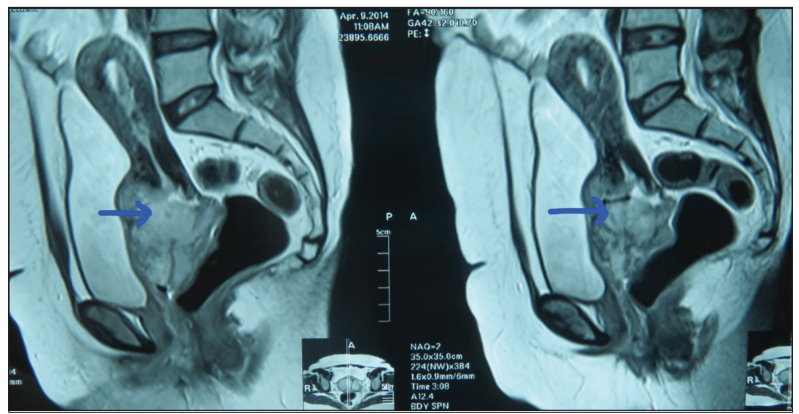

Figure 1: Magnetic resonance imaging pelvis (sagittal view) showing the lesion of malignant melanoma in the cervix (arrow)

bladder with loss of intervening fat planes. Posterior, the mass was focally infiltrating into the anterior rectal wall [Figure 1]. Computed tomography scan chest/abdomen was negative for metastases. Disease was staged as International Federation of Gynecology and Obstetrics (FIGO) Stage IVA if staged on the lines of squamous cell carcinoma cervix.

The case was discussed in the city tumour board of Karachi. The patient refused surgery due to the risk of pelvic exenteration. Immunotherapy with ipilimumab was not feasible due to high cost. The option of radiotherapy was not considered due to the risk of rectovaginal fistula formation. Combination chemotherapy such as Cisplatin, Vinblastine and Dacarbazine was not considered due to a higher risk of toxicity with no survival benefit over the single agent. The patient was planned for six cycles of single agent Dacarbazine therapy $\left(250 \mathrm{mg} / \mathrm{m}^{2}\right)$ day $1-5$ Q4 weeks. However, the patient only received only three cycles and quit treatment.

\section{Discussion}

Malignant melanoma is a rare malignancy of skin. It accounts for only $2 \%$ of all skin malignancies. Predisposing factor is genetics, sun exposure, and multiple nevi. Prognosis of these case is usually poor if involving nodes or metastasizing to a distant site. Most common site of metastasis is a lung. Most common mucosal sites of involvement are head and neck followed female genital tract regions..$^{[4]}$ No definite treatment is available if the mass is irresectable. Most of the dermal melanoma are resectable, but this was a rare case where mass was at an unusual site and irresectable. It was irresectable due to its invasion into surrounding structures. Mucosal melanoma usually behaves aggressively. Their spread to a regional lymph node is quite early as compared to dermal melanoma and makes the condition of patient miserable if not resected well in time.

We reviewed the literature to check the total number case of cervical melanoma reported up till now. A total of 60 cases have been reported from different part of the world.$^{[5]}$ The latest report found was from Portugal published in 2011. ${ }^{[6]}$

\section{Conclusion}

Primary malignant melanoma of the cervix is rare and requires multidisciplinary management.

\section{Conflict of Interest}

The authors declare that they have no conflict of interest.

\section{References}

1. Saika K, Sobue T. Cancer statistics in the world. Gan To Kagaku Ryoho 2013;40:2475-80.

2. Baxter AJ, Hughes MC, Kvaskoff M, et al. The Queensland study of melanoma: Environmental and genetic associations (Q-MEGA); study design, baseline characteristics, and repeatability of phenotype and sun exposure measures. Twin Res Hum Genet 2008;11:183-96.

3. Ahmed A, Alam MB, Khan W, et al. Frequency and characteristics of skin cancers diagnosed at Ayub medical college, Abbottabad Pakistan from 1995-2003. J Ayub Med Coll Abbottabad 2007;19:3-6.

4. Chang AE, Karnell LH, Menck HR. The national cancer data base report on cutaneous and noncutaneous melanoma: A summary of 84,836 cases from the past decade. The American college of surgeons commission on cancer and the American cancer society. Cancer 1998;83:1664-78.

5. Calderón-Salazar L, de Leon DC, Montiel DP, et al. Primary malignant melanoma of the uterine cervix treated with ultraradical surgery: A case report. ISRN Obstet Gynecol 2011;2011:683020.

6. Simões M, Cunha V, Nabais H, et al. Primary malignant melanoma of the uterine cervix--case report and review. Eur J Gynaecol Oncol 2011;32:448-51. 